\title{
Correlations between Forging Tool Lifetime and the Continuous Curvature Transitions
}

\author{
F. Tancsics ${ }^{1}$, T. Ibriksz ${ }^{1}$ \\ ${ }^{1}$ Széchenyi István University, Department of Material Sciences and \\ Technology, 9026 Győr, Egyetem tér 1, Hungary \\ e-mail: tancsics@sze.hu
}

Abstract: The forged work-piece geometry is determined by different standards and bilateral specifications, so the geometry is not allowed to be changed by the technologist according to their own decision. Therefore, the lifetime of the tool which gives the final geometry is a key issue. The tool lifetime is mainly influenced by technology and process parameters. Regarding these parameters, there are many classical optimization solutions available to increase tool life. In our work, we have looked for a technical device that can complement classic solutions to further enhance the effectiveness in the field of forging tools lifetime. In our opinion, such a technical device may be a continuous curvature of the surface transitions at the preformed geometry. In our work, we investigated the effect of the continuous curvature for a function reducing the forging work at a real geometry.

Keywords: forging; tool lifetime; B-spline; forging force

\section{Introduction}

Connection between tool surfaces, which take effect on material flow, can be designed with continuity in the connecting points. This continuity is mostly achieved by connecting the tangential of the two endpoints. These endpoints ensure the $C^{1}$ continuity in common points of the curves. The result of relationship is the rounding radius, which provides a smooth surface transition. Such a transition is acceptable in general for most applications because of the favourable material flow, minimized wear rate and low forging energy [1][2].

This continuity can be ensured by higher-level connections driven splines as well, which are commonly used in other engineering applications as well [3][4]. An example of this is the connection with continuous curvature between certain surface 
elements with $C^{2}$ continuity. This $C^{2}$ continuity simultaneously provides the criterions of tangential and continuous curvature at the connecting endpoints of curves. In between the endpoints the curvature might constantly change. In fact, this change can be expressed as an angle, which is the degree of the change of the tangential vector relative to the arc length in the instantaneous point on the curve. Therefore, at the connection of a straight and a spline, the initial curvature of the spline should be zero, consequently, the first elementary section of the spline is a straight. This type of curvature may provide smoother transition between surfaces regarding material flow. These latter connection forms were tested on a real preformed and finished 3D forging tool geometry of crown wheel (Fig. 1).

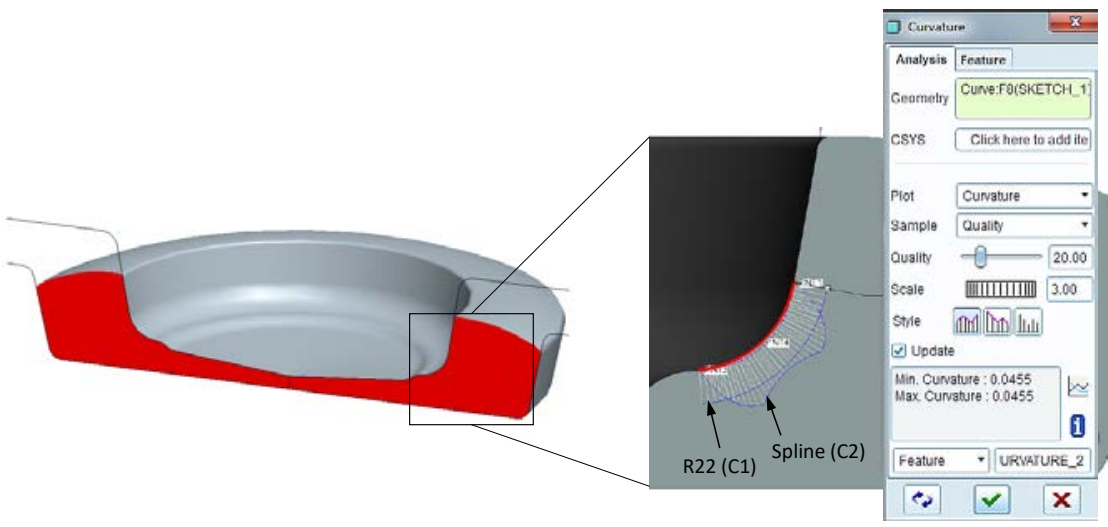

Figure 1. The preformed crown wheel (left) and the curvatures of inspected area (right) are shown with highlighted curvature of $R 22$ radius

In Fig. 1 left a simple forged crown wheel preformed geometry can be seen whereon our concept can be well illustrated. The inspected radius in Fig. 1 right, which is indicated by dark loop, is R22 $\mathrm{mm}$ and its curvature is constant (0.0455). The radius is connected tangentially to the other sections on the profile curve. In these endpoints the radius value is infinite, and the value of the curvature is zero. Therefore, the curvature in these points is not continuous. Fig. 1 shows a spline curve as well, with different curvature radius. There is $C^{2}$ continuity at the endpoints of spline curve and between the endpoints there is a continuously variable curvature. The question, which of the available spline curves can be more appropriate for replacing the radius, arises. In our point of view, we only need to ensure that the $C^{2}$ continuity at the endpoints of spline curve meets and the approximation curve method is used between these points.

This requirement is practically equivalent to usage of cubic B-spline curves [5]. The Pro/E CAD system, similarly to other market leader CAD systems, uses the 
NURBS (Non Uniform Rational B-Spline) curves for complex tasks [6]. This type of curve can be simplified according to the curve classes bound together with wellknown rational B-splines [7]. Based on these considerations the Pro/E CAD system is a suitable application for function analysis [8]. The selected spline system (in other words: "control point spline method") connects to the sections of the profile curve by continuous curvature and it is locally changeable. The local curvature of the spline curve can be controlled by one or more treadpoints (point on the curve). In Fig. 2 the complete curvature of spline curve can be controlled by three control points or by control polygon (points on the purple lines). It is worth considering to optimize the number of control points to achieve the most even curvature.

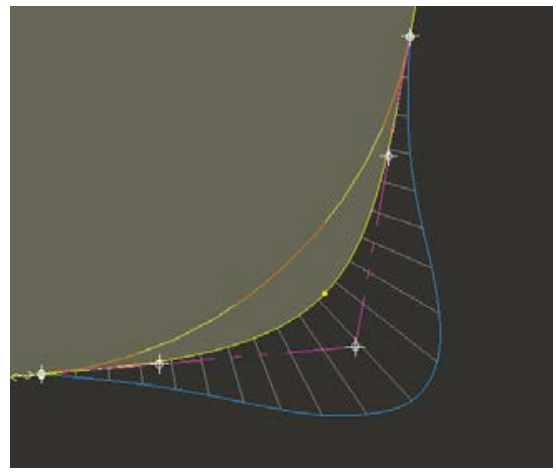

Figure 2. The variable curvature between the endpoints of spline curve

The geometry can be freely variable within certain limits, however, the volume constancy, referring to the initial volume, must be ensured. These variable geometries considering the volume constancy can be ensured with limited extreme value calculation in different CAD solution [9] [10]. Based on volume constancy, the usage of transitions with continuous curvature may be accomplishable as well. We can also specify a maximum allowable curvature (boundary) and we can determine the suitable local position of the control point with limited extreme value calculation. In our point of view, the maximum allowable curvature should be determined based on the radius of the finished geometry. With respect to the modified surface transitions, the Simufact.forming finite element software is suitable for determining which preforming has the less energy demand. This software is a member of Computational Fluid Dynamics (CFD) software family, so in this case it can provide adequate analyses about the incompressible continuum flows along to modified tool surfaces to determine the energy of material motion using numerical approaches. 


\section{FEM testing on the new surface transition}

Our primary goal was to find a detectable decrease in deformation energy or forging force. If it is possible, this value can be optimized later, so the main goal will be available. We produced different spline curves with different curvature between the examined endpoints of the tool surface in the Pro/E CAD system. We wanted to know if we may get a more suitable geometry in terms of material flow instead of the original radius connection. The new geometry is more appropriate for material flow, which reduces the deformation energy and does not influence negatively the flow conditions considered in Ref. [11]. For the required finite element analysis we used the Simufact.forming CAE system (hereinafter referred to as "SF").

\subsection{Investigating the lower forging energy}

For simulation test we used a maxima crank press equipment with $80 \mathrm{MN}$ nominal force. For the forging technology (e.g. forging temperatures, tact time, material grade, etc.) and tools (e.g. 3D models, surface hardening and roughness, etc.) we used the technological parameters given by Rába engineers. The forging process consists of three steps, upsetting (for descaling) - preforming - finishing, thus we used a multi-stage technology method in the simulation. Except for some preprocess data, the technology cannot be disclosed. The approved pre-process data for publishing, which are enough to understand the energy analysis of the effect of continuous curvature, are the following:

- Imported file formats from CAD system: DXF (Drawing eXchange Format) file formats to simplify the simulation geometry of forging tools

- Selected options for analysis in SF system: Hot forging, 2D axisymmetric, solver type is FE (Finite Elements)

- $\quad$ The mesh type is AFQ (Advancing Front Quad)

- The element edge is $1.5 \mathrm{~mm}$

- The HTC (Heat Transfer Coefficient) value between the work piece and the environment is $50 \mathrm{~W} / \mathrm{m}^{2} \mathrm{~K}$, the same as between the forging tools and the environment,

- Forging temperature is $1100{ }^{\circ} \mathrm{C}$, the average tool temperature is $200{ }^{\circ} \mathrm{C}$

- Tool elasticity assign is: Rigid

- Materials selected from the library of SF: for forging tool it is 55NiCrMoV6 material grade, and for forging piece it is $20 \mathrm{MnCr} 5$ material grade with their mechanical, technological and chemical properties. 
- The friction mechanism between the tool and the workpiece was approximated by the constant value of Kudo's friction number. This value was set to 0.45 based on the previous tribological studies.
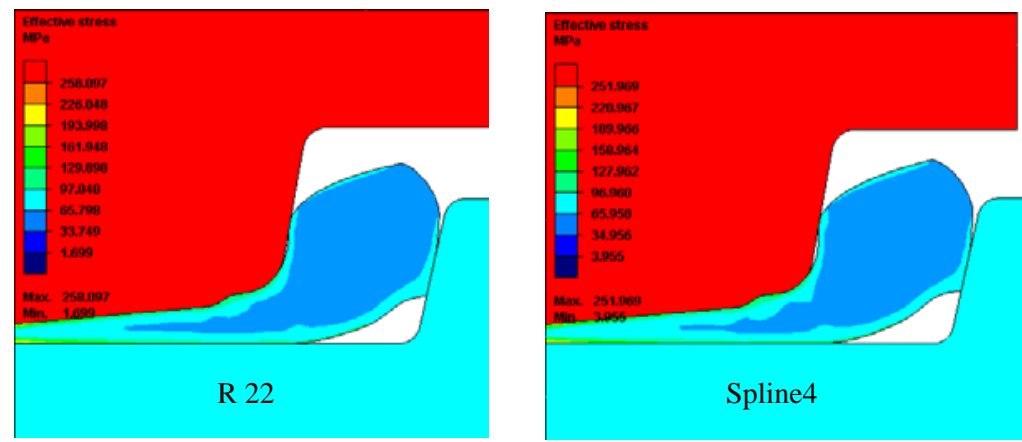

Figure 3. Normal stress distribution differences in case of radius (R22) and spline4 curve in SF system

Fig. 3 shows a simulation result of the normal stress distribution of workpiece in connection with the rounding radius and one of the spline curves. We can state that the acting normal stress on the surface of the tool in the test environment is reduced by Spline 4 curve (see Table 1). In addition, it is visible that the work-piece surface is separated from the top tool surface. This effect was typical of all types of examined connecting curves. From this, it can be concluded that the lower part of the connecting curve has a strong effect on the flow of the material. It also means that the distribution of the compression tension in direction of corner radius of the cavity has a higher intensity. This indicates more favorable material flow conditions for filling the cavity. The results in details of the analysis are given in Table 1.

Table 1. Data of forging in case of different curves based on the SF analysis

\begin{tabular}{|c|c|c|c|c|}
\hline \multirow{2}{*}{ Name } & $\begin{array}{c}\text { Forging } \\
\text { process }\end{array}$ & $\begin{array}{c}\text { Deformation } \\
\text { energy [KJ] }\end{array}$ & $\begin{array}{c}\text { Deformation } \\
\text { force [MN] }\end{array}$ & $\begin{array}{c}\text { Total energy } \\
{[\text { [KJ] }}\end{array}$ \\
\hline \multirow{2}{*}{$\begin{array}{c}\text { reference } \\
\text { (radius) }\end{array}$} & preforming & 604.565 & 18.324 & \multirow{2}{*}{1101.993} \\
\cline { 2 - 4 } & finishing & 497.428 & 78.05 & \\
\hline \multirow{2}{*}{ Spline2 } & preforming & 613.536 & 18.708 & \multirow{2}{*}{1113.129} \\
\cline { 2 - 4 } & finishing & 499.593 & 77.562 & \multirow{2}{*}{1140.577} \\
\hline \multirow{2}{*}{ Spline3 } & preforming & 627.846 & 18.839 & \multirow{2}{*}{1108.375} \\
\cline { 2 - 4 } & finishing & 512.731 & 77.346 & \\
\hline \multirow{2}{*}{ Spline4 } & preforming & 597.472 & 17.789 & \multicolumn{2}{|c}{} \\
\cline { 2 - 4 } & finishing & 510.903 & 78.626 & \\
\hline
\end{tabular}


The energy data for the simulations are summarized in tables. These data represent the maximum values at the end of the stroke. As we only need this data, we disregard the shape of the energy diagrams. The same consideration refers to force-related values as well. From the data in Table 1, it can be concluded that the needed preforming energy and the contact force were reduced together in case of Spline4 [12]. On the other hand, this advantage is lost at finishing forging.
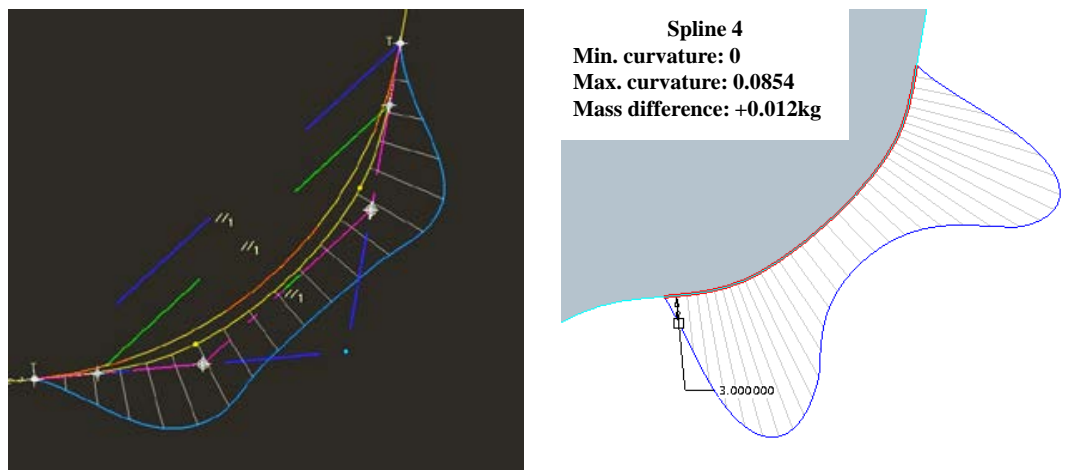

Figure 4. The symmetrical curvature of spline4 curve with two treadpoints

Fig. 4 shows spline4, which provides the local curvature by two treadpoints. Hereinafter, this type of curve is worth examining and this geometry is worth being developed. The difference from the initial mass based on the limited extreme value optimization is $+0.012 \mathrm{~kg}$. This weight difference can be reduced later. Two conclusions can be drawn in connection with the continuation of examination. On the one hand, there is a spline curve that reduces deformation force and energy. On the other hand, the analysis should also be extended to the finishing process, and the task should be solved in a complex way. We found a suitable curve geometry to reduce deformation energy so our primary goal is accomplished. In addition, we came to the conclusion that the spline 4 curve is worth being further examined.

\subsection{Complex examination to decrease the forging energy}

In further to reach better results, we should preserve the control point spline method because it guarantees well-controlled positions in the CAD system. The start and end points of spline 4 curve coincide with the radius endpoints. Due to preserving $\mathrm{C}^{2}$ continuity, the points of the spline curve are located on the common tangent crossing through the radius endpoints. If we take 4 control points on these 2 tangents, only 2 variable dimensions have to be changed in a limited extreme value calculation in case of a symmetrical spline (Fig. 4). It is worth examining spline4 curve with asymmetric curvature as well. 
In this case three dimensions have to be changed for a limited extreme value calculation. The result of this calculation can be seen in Figure 5. Additionally, it can be seen that approximation of volume constancy has also been improved.
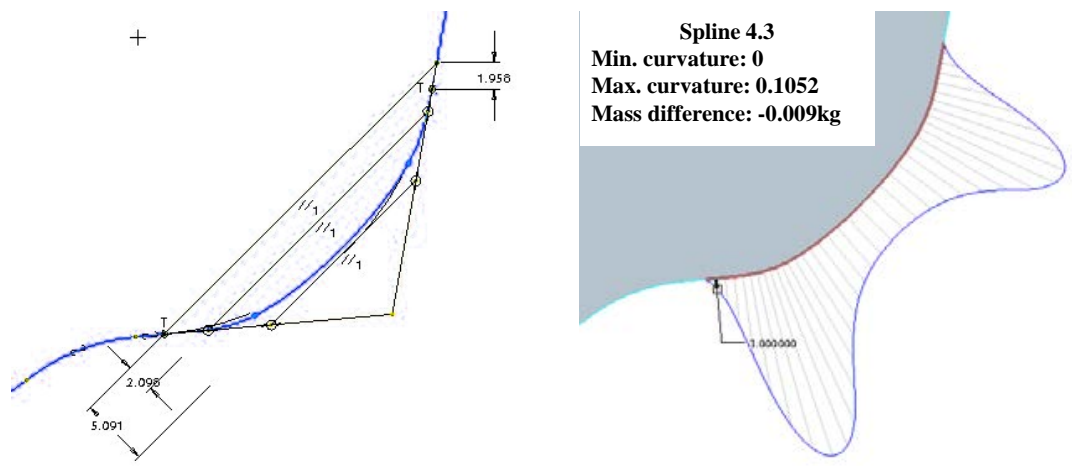

Figure 5. The optimized asymmetrical curvature of spline4.3 curve

The code number 4.3 refers to the 3 variables of the asymmetric curvature of Spline4 curve. In the following section, we examined this type of curve because of better approximation of geometry and greater variation possibilities. However, using multiple variables can result in complicated optimization algorithms as well. Keeping this in mind, our optimization algorithm has been simplified according to the principle of dynamic programming [13]. We have used the minimum values of deformation energy for additional examination in all cases.

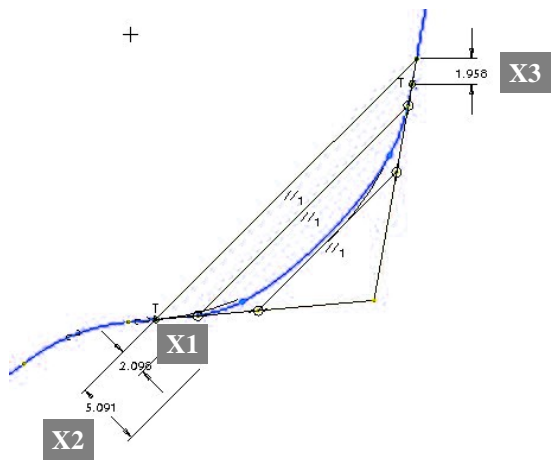

Figure 6. The variable dimensions of the spline4.3 curve

This time with the help of the variables, the new goal is to find the minimum value of deformation work by changing the dimensions (Fig 6). Dimensions X1 and X3 are freely variable. Dimension X2 is already limited by the change in dimension X1. 
Based on these conditions, we examined the deformation work in SF system by changing the dimension of $\mathrm{X} 1$ and $\mathrm{X} 3$. The volume constancy is ensured by parameter X2. With the created curves, the average mass deviation varied around $0.0003 \mathrm{~kg}$. Consequently, the approximation accuracy of the initial mass improved further as well. This approximation accuracy is an already acceptable value. The spline curves named by numbers of the variants. For example, if X1=2 $\mathrm{mm}$ and $\mathrm{X} 3=1$ $\mathrm{mm}$, the code of spline curve is Spline2.1.
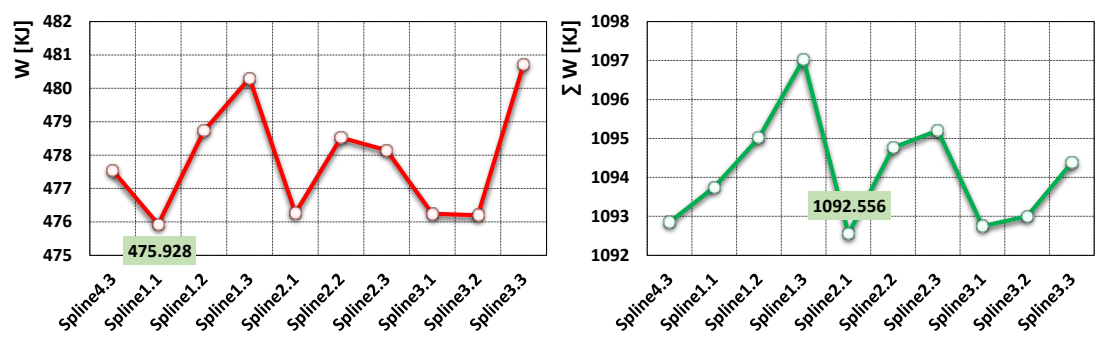

Figure 7. The deformation energy minimum values regarding the finishing forging and the total deformation based on the SF analysis

The variations of range of function were plotted on charts of energy requirements as a function of the final deformational and total energy demands (Fig 7.). Analyzing these diagrams, it can be stated, that Spline2.1 curve has a minimum value on the total energy demand diagram and has an extreme low value on the final deformational diagram. So, this spline curve is worth dealing with.

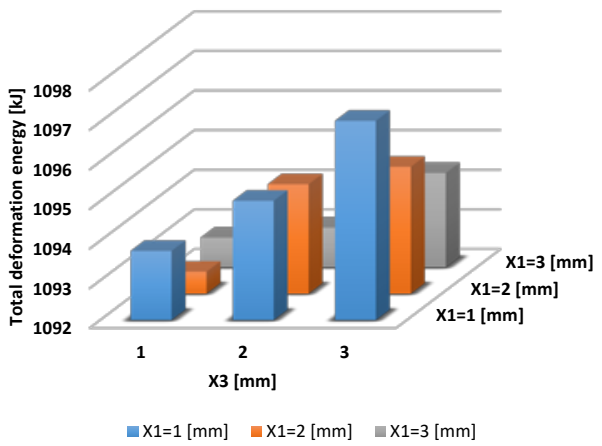

Figure 8. The total energy demand in case of different types of spline4.3 curves based on the SF analysis 
The minimum value of total energy demand is shown in a special column diagram as well (Fig. 8). Further energy reduction is possible by improving Spline2.1 curve (Fig 9.).

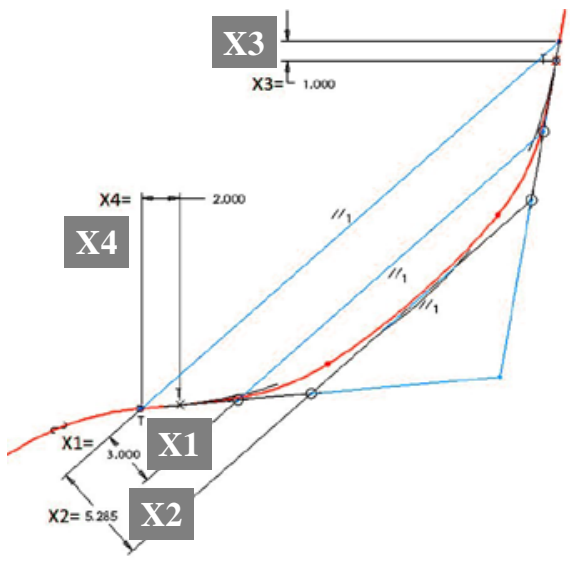

Figure 9. Introducing a new variable (X4) to improve spline2.1 curve

For this we need to introduce a new variable and establish new relationships between the variables. In this relation-system, dimensions X1, X3 and X4 are freely variable, while dimension $\mathrm{X} 2$ is limited by dimension $\mathrm{X} 1$.

According to the previously applied principle, we examined the energy values by changing dimensions $\mathrm{X} 1, \mathrm{X} 3$ and $\mathrm{X} 4$. As before, the volume constancy is ensured by parameter $\mathrm{X} 2$. In the examined geometric context we have to formulate the boundary conditions, which are the following:

$$
\begin{gathered}
0 \leq \mathrm{X} 1<\mathrm{X} 2, \\
0 \leq \mathrm{X} 3, \\
0 \leq \mathrm{X} 4<2.093 .
\end{gathered}
$$

Whereabouts: X2 follows from volume constancy, insofar if the value of X4 is greater than 2.093, it would already be defined with another radius, so setting the continuity of the curvature may become complicated. With the created curves, the average mass deviation is alike with Spline2.1, so it is an acceptable value.

We named the spline curves based on the number of variants, as we mentioned it before. For example, if X1=3 mm, X3=1 mm and X4=2 mm, the code of spline curve is Spline3.1.2. 

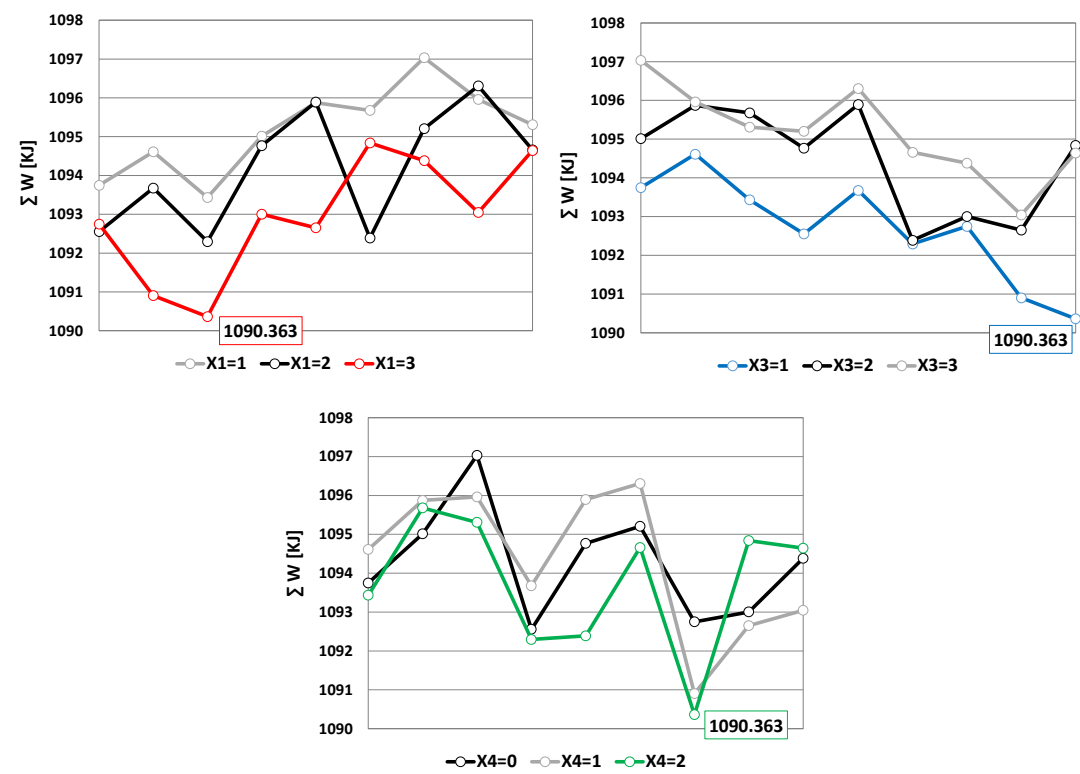

Figure 10. Seeking for spline curve of minimum energy based on the SF analysis

Performing the test for all variations of curves may lead to a dataset that is hard to handle. This dataset should be interpreted purposefully for the effects of each parameter. We continue to apply the principle of dynamic programming as we previously mentioned. From the combination of the minimum curves, the code of the minimum spline curve can be generated, which is Spline3.1.2 curve (Fig. 10). If the dataset is manually processed, we can get the same result. Based on the results of examination, we may state the last simplifying conditions.

- $\mathrm{X} 1$ should be increased as long as possible. In the extreme case, the value of X1 approximates X2 with an integer. Value X2 is determined by the law of volume constancy.

- $\quad$ X3 should be set as low as possible. In terms of integers, it is advisable to set X3 to one or zero.

- $\quad$ X4 should be set to maximum integer.

Based on the above findings, the last test was done with the following settings:

$$
\begin{gathered}
0 \leq \mathrm{X} 1 \sim \mathrm{X} 2=4, \\
0 \ldots 1=\mathrm{X} 3,
\end{gathered}
$$




$$
\mathrm{X} 4=2 .
$$

The results in details are summarized in Table 2 below.

Table 2. Data of energy in case of different spline types based on the SF analysis

\begin{tabular}{|c|c|c|c|}
\hline \multirow{2}{*}{ Name } & $\begin{array}{c}\text { Forging } \\
\text { process }\end{array}$ & $\begin{array}{c}\text { Deformation } \\
\text { energy [KJ] }\end{array}$ & $\begin{array}{c}\text { Total energy } \\
\text { [KJ] }\end{array}$ \\
\hline \multirow{2}{*}{ Spline4.0.2 } & preforming & 620.882 & \multirow{2}{*}{1089.96} \\
\cline { 2 - 3 } & finishing & 469.078 & \\
\hline \multirow{2}{*}{ Spline4.1.2 } & preforming & 614.646 & \multirow{2}{*}{1090.047} \\
\cline { 2 - 3 } & finishing & 475.401 & \\
\hline
\end{tabular}

From the data in Table 2, it can be concluded that the needed settings for giving the minimum summarized forging energy in the overall formula are the following:

$$
\begin{gathered}
\mathrm{X} 1 \sim \mathrm{X} 2, \\
0=\mathrm{X} 3, \\
\mathrm{X} 4=\max .
\end{gathered}
$$

The curve refinements can also be carried out in the form of factionary variables, but no substantial change is possible. Depending on the total work and the refinement of curvature continuity, there is a rational limitation point of energy reduction. Fig. 11 shows this limited relationship between the reducible energy and curvature continuity.

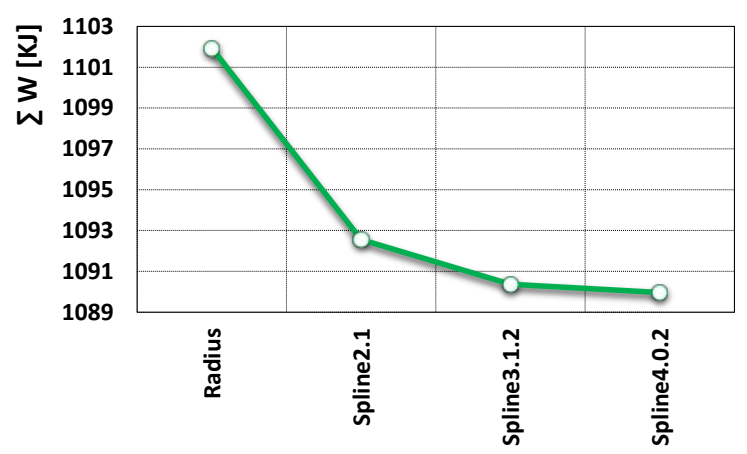

Figure 11. The cumulative forging energy reduction achieved by dynamic programming method. 
The curvature of Spline4.0.2 curve is shown in Figure 12. It is worth observing the shape of the curvature which is more favorable for material flow. This geometry has a more favorable effect on sliding systems of metals.

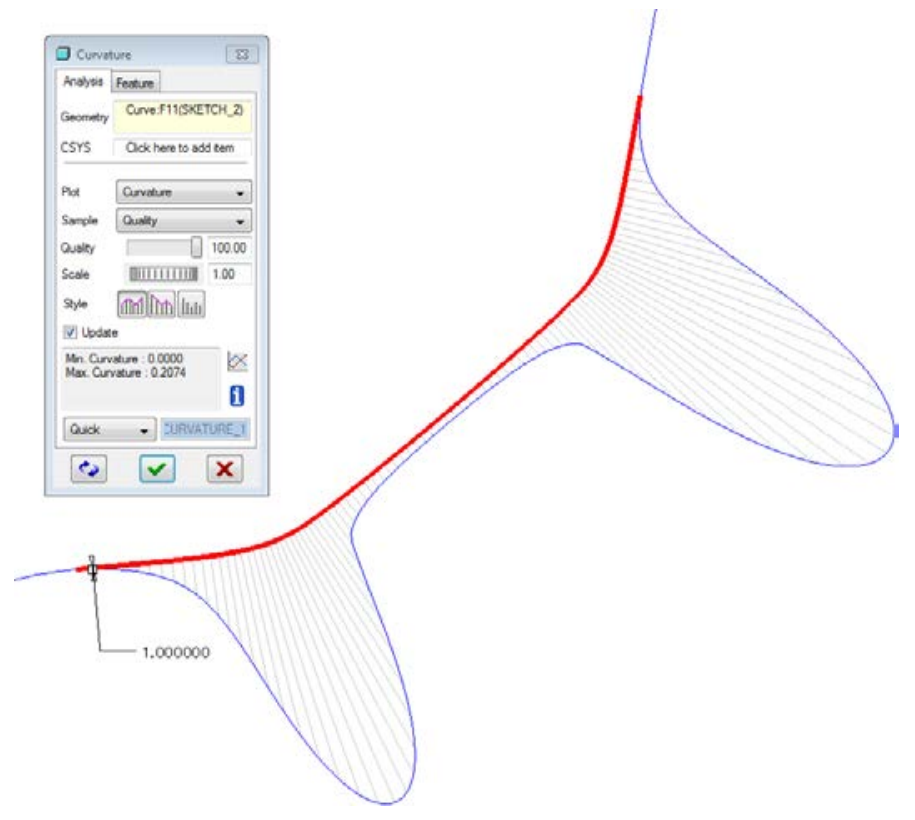

Figure 12. The curvature of Spline4.0.2 curve

For this we need to introduce a new variable and establish new relationships between the variables. In this relation-system, dimensions X1, X3 and X4 are freely variable, while dimension $\mathrm{X} 2$ is limited by dimension X1.

Finally, from the data in Table 2, we selected the Spline4.02 curve of the preforming tool and examined the effect of the new geometry on distribution of effective strain rate. This parameter is a good indicator of the state of material flow and filling of cavity. The picture at the top side of Figure 13 is showing the contour line of the original radius of upper tool and the flow lines of material flow in SF system. The picture below it shows the connection with optimized continuous curvature of upper tool and the flow lines of the material flow. The effective strain rates are displayed on the left side of both images. When looking at the pictures, our first impression is that there is no difference between them. Taking a closer look at the distribution of local deformations around the flow lines, the bottom image shows a more even distribution and sometimes smaller value. 
All this leads to a slight decrease in energy and a better filling in cavity. Both analyses were made with the same pre-process settings.
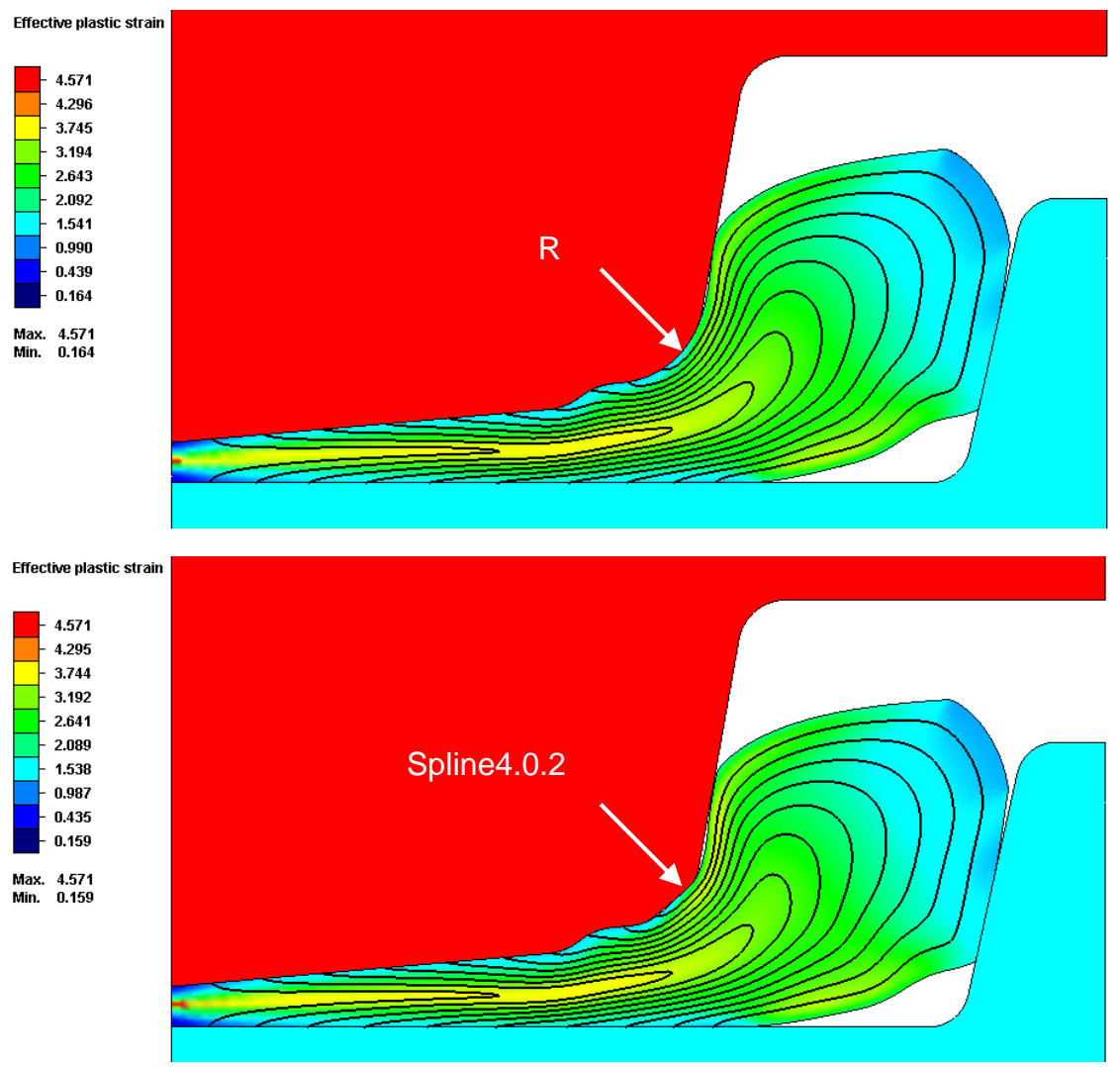

Figure 13. Differences between the original radius and the curvature optimized for the material flow based on the SF analysis

\section{Summary and discussion}

In our work we investigated whether the surfaces with continuous curvature transitions affect the energy demand of the forging. We proved the existence of this effect, the magnitude of which was about $1 \%$ for a given radius. In the first approach, this does not seem to be a great value, but it should not be neglected in case of the radius which most exposed to wear impacts. If the hardness of tools increases by any hardening process, the effect of the curvature may be much stronger as well [14]. 
We should remember that we only tested one average radius dimension. The effect can also be increased in case of multiple or larger radii. Therefore, our next goal is to investigate the real impact of the presented virtual connection in manufacturing conditions. Summarizing our work, the main goals are fulfilled basically for industrial applications, so the next important step may be the practical adaptation.

\section{Acknowledgement}

The paper was written with the support of the project titled "Internationalization, initiatives to establish a new source of researchers and graduates and development of knowledge and technological transfer as instruments of intelligent specializations at Széchenyi István University" (project number: EFOP-3.6.1-16-2016-00017). We would also like to thank Ernő Halbritter PhD and Ádám Futácsi for assisting in connection with the theoretical background of this article.

\section{References}

[1] L. Shen, M. Bellus, Numerical Simulation of Multistep Warm Extrusion Forming for the Outer Race of Universal Joint, Journal of Engineering Science and Technology Review 11 (6) (2018) pp. 162-168. doi: $\underline{10.25103 / \text { jestr.116.20 }}$

[2] Y. Chen, Preform Design for Forging and Tube Hydroforming Processes, Ph.D. thesis, Graduate Faculty of North Carolina State University, Department of Mechanical Engineering, (2011).

[3] F. Hajdu, Gy. Molnárka, Parallelization of Numerical Examination of Nonlinear Systems using Maple, Proceedings of the $5^{\text {th }}$ International Conference on Parallel, Distributed, Grid and Cloud Computing for Engineering Dun Eaglais, CCP:111, paper 30, Stirlingshire, UK, (2017).

[4] F. Hajdu, P. Mika, P. Szalai et al., Determination of Vibrations Caused by the Road Profile in Case of a Fire Truck, International Advanced Research Journal in Science, Engineering and Technology 6 (3) (2019) pp. 52-58. doi: $\underline{10.17148 / \text { iarjset.2019.6310 }}$

[5] E.U. Agom, F.O. Ogunfiditimi et al., Cubic B-Spline Collocation and a Domain Decomposition Methods on $4^{\text {th }}$ Order Multi-point Boundary Value Problems, Journal of Advances in Mathematics and Computer Science 27 (1) (2018) pp. 1-7. doi: $\underline{10.9734 / j a m c s / 2018 / 40656}$ 
[6] E. Unvert, P. Atkinson, D.J. Tancock, Applying 3D Scanning and Modeling in Transport Design Education, Computer-Aided Design \& Applications 3 (14) (2006) pp. 41-48.

doi: $\underline{10.1080 / 16864360.2006 .10738440}$

[7] B.T. Bertka, An Introduction to Bezier Curves, B-Splines, and Tensor Product Surfaces with History and Applications, 1st Edition, University of California, Santa Cruz, (2008).

[8] J. Wronecki, Surface Modelling Techniques for Automotive and Product Design, 1st Edition, East Tennessee State University, American Society for Engineering Education, (2007).

[9] F. Tancsics, Development and Practical Implementation of Basically Computer-Aided Design Methods of Virtual Forging Technologies, Ph.D. thesis, Széchenyi István University, MMTDI, (2013) (in Hungarian)

[10] F. Tancsics, T. Gergye, E. Halbritter, Using up-to-date softwares in technological improvements and optimisation of multiple cavity forging, Strojnicky Casopis, Journal of Mechanical Engineering 62 (4) (2011) pp. 191-203.

[11] L. Shih-Hsien, U.C. Chai et al., Generalized Optimizations of Two-Stage Forging of Micro/Meso Copper Fastener, MATEC Web of Conferences 185 (00002) (2018) p. 11.

doi: $10.1051 /$ matecconf/201818500002

[12] N. Fois, M. Watson et al., An Investigation of the Relationship between Wear and Contact Force for Abradable Materials, Journal of Engineering Tribology 229 (2) (2015) pp. 136-150.

doi: $\underline{10.1177 / 1350650114545139}$

[13] V.L. Matos, A.B. Philpott, E.C. Finardi, Improving the Performance of Stochastic Dual Dynamic Programming, Journal of Computational and Applied Mathematics 290 (2015) pp. 196-208.

doi: $\underline{10.1016 / \text { j.cam.2015.04.048 }}$

[14] D.C. Ko, D.H. Kim, B.M. Kim, Finite Element Analysis for the Wear of TiN Coated Punch in the Piercing Process, Wear 252 (11-12) (2002) pp. 859869.

doi: 10.1016/S0043-1648(02)00032-7 